J. theor. Biol. (2002) 218, 309-321

doi:10.1006/yjtbi.3078, available online at http://www.idealibrary.com on IDE $\mathbf{L}^{\circledR}$

\title{
Stochastic vs. Deterministic Modeling of Intracellular Viral Kinetics
}

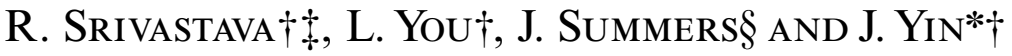

$\dagger$ Department of Chemical Engineering, University of Wisconsin, 3633 Engineering Hall, 1415 Engineering Drive, Madison, WI 53706, U.S.A., \$McArdle Laboratory for Cancer Research, University of Wisconsin Medical School, Madison, WI 53706, U.S.A. and §Department of Molecular Genetics and Microbiology, University of New Mexico School of Medicine, Albuquerque, NM 87131, U.S.A.

(Received on 5 February 2002, Accepted in revised form on 3 May 2002)

Within its host cell, a complex coupling of transcription, translation, genome replication, assembly, and virus release processes determines the growth rate of a virus. Mathematical models that account for these processes can provide insights into the understanding as to how the overall growth cycle depends on its constituent reactions. Deterministic models based on ordinary differential equations can capture essential relationships among virus constituents. However, an infection may be initiated by a single virus particle that delivers its genome, a single molecule of DNA or RNA, to its host cell. Under such conditions, a stochastic model that allows for inherent fluctuations in the levels of viral constituents may yield qualitatively different behavior. To compare modeling approaches, we developed a simple model of the intracellular kinetics of a generic virus, which could be implemented deterministically or stochastically. The model accounted for reactions that synthesized and depleted viral nucleic acids and structural proteins. Linear stability analysis of the deterministic model showed the existence of two nodes, one stable and one unstable. Individual stochastic simulation runs could access and remain at the unstable node. In addition, deterministic and averaged stochastic simulations yielded different transient kinetics and different steady-state levels of viral components, particularly for low multiplicities of infection (MOI), where few virus particles initiate the infection. Furthermore, a bimodal population distribution of viral components was observed for low MOI stochastic simulations. The existence of a low-level infected subpopulation of cells, which could act as a viral reservoir, suggested a potential mechanism of viral persistence.

(C) 2002 Elsevier Science Ltd. All rights reserved.

\section{Introduction}

Viruses are ideal systems for probing how the static linear information encoded by a genome defines a dynamic nonlinear process of growth and development. Their genomes are small, ranging in size from 3 to 300 kilobases, and in many cases their encoded functions are genetically, biochemically, and structurally

\footnotetext{
*Corresponding author. Tel.: + 1-608-265-3779; fax: $+1-608-262-5434$.

E-mail address: yin@engr.wisc.edu (J. Yin).
}

well-characterized. We have developed models for the intracellular growth of bacteriophage T7 (Endy et al., 1997; You \& Yin, 2001) and HIV-1 (Reddy \& Yin, 1999), which have enabled us to suggest novel anti-viral strategies (Endy \& Yin, 2000), visualize dynamic patterns of genetic regulatory elements (You \& Yin, 2000), and probe how virus growth depends on genome organization (Endy et al., 2000) and host resources (You et al., 2002). To date, we have formulated our viral models as coupled ordinary differential equations and algebraic expressions that employ parameters from the literature and 
are numerically integrated to reveal dynamic behavior.

Here, we seek to better understand what differences may result from stochastic and deterministic kinetic approaches to modeling viral kinetics. A virus infection may be initiated by a single virus particle that delivers its genome, a single molecule of DNA or RNA, to its host cell (Jilbert et al., 1996). Under such conditions, the inherently stochastic nature of the ensuing processes may give rise to dynamics that differ significantly from those predicted by deterministic models. There are many examples of stochastic biological models in the literature (Chin \& Willsky, 1989; McAdams \& Arkin, 1997; Arkin et al., 1998; Goss \& Peccoud, 1998; Srivastava et al., 2001), as well as examples comparing deterministic and stochastic kinetics of simple biochemical systems (Érdi \& Tóth, 1988), and linear and nonlinear chemical systems (Kurtz, 1972; Zheng \& Ross, 1991). A nonlinear model of the intracellular kinetics of a generic non-lytic virus was developed. For a non-lytic virus, the level of intracellular viral components must be regulated in some manner to avoid destroying the cell simply via excess propagation. The question of how the levels of components, particularly the ones existing at low levels, are regulated in the face of stochastic fluctuations is an intriguing one. The current computational study showed differences in stochastic and deterministic implementations of the viral model. It also showed how a low-level viral reservoir may be established, suggesting a potential mechanism for viral persistence.

\section{Model Development}

A simple network of a model virus, shown in Fig. 1, was developed to explore differences between stochastic and deterministic model implementations. The components studied were the viral nucleic acids and a viral structural protein (struct). The viral nucleic acids were classified as genomic (gen) or template (tem). The genome, whether it is DNA, positive-strand RNA, negative-strand RNA, or some other variant, is the vehicle by which viral genetic information is transported. The genome can undergo one of the two fates. The first possibility is that it may be modified, whether through integration into the host genome or some other type of processing (reverse transcription, etc.), to form a template; the template refers to the form of the nucleic acid that is transcribed and involved in catalytically synthesizing every viral component. The second possibility for the genome is that it may be packaged within structural proteins to form progeny virus; structural proteins may be capsid proteins or envelope proteins.

The standard sequence of viral replication events involves the amplification of the viral template after the infection, followed by production of progeny virus. DNA viruses, for example, initially make low levels of non-structural

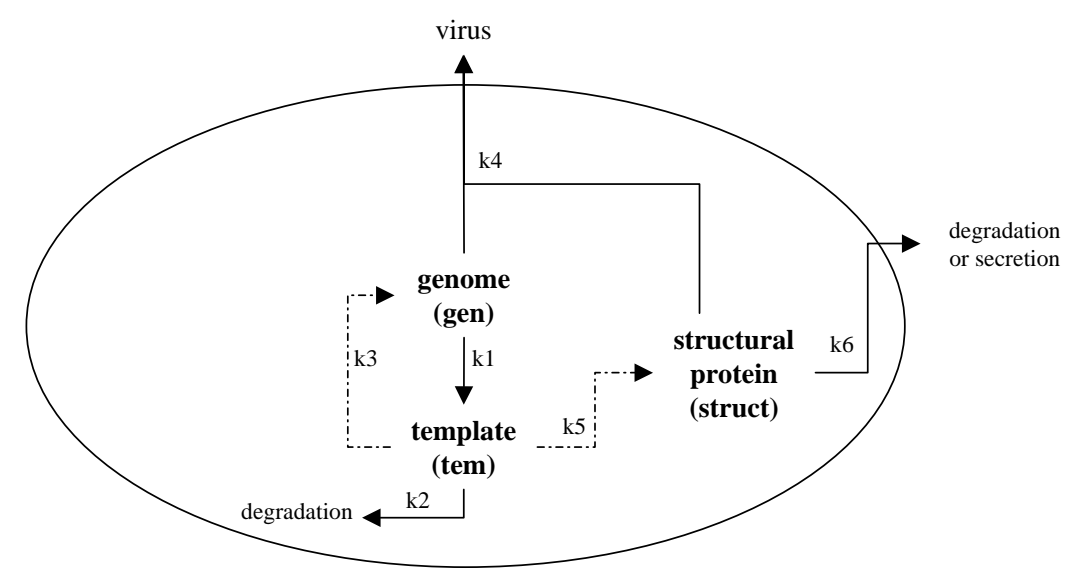

FIG. 1. Model of viral replication cycle. The model tracks template (tem), genome (gen), and structural protein (struct). The tem is only used catalytically in the synthesis of gen and struct. The catalytic reactions are represented by (-----) lines. 
proteins that catalytically amplify the number of template molecules. Once the number of template molecules is sufficiently high, structural proteins are synthesized for incorporation into progeny particles. The timing of replication events may be tied to external factors, as illustrated by the dependence of human papilloma virus replication on the differentiation state of its host cell (Knipe \& Howley, 2001). In our model, the switch from viral genome amplification to virus production was regulated through viral mass action kinetics, as was shown through the simulation studies. Because the switch could mathematically be accomplished solely through the dynamics of key viral components, for our purposes it was unnecessary to introduce any explicit dependence upon the host. However, an implicit host dependence is reflected in the values of the kinetic parameters of the model. For example, if in a given host cell a certain amino acid is limiting, it may be reflected by a lower rate constant for protein synthesis.

The modeling network employed was lumped, in the sense that many individual reaction steps were combined into a single step. For example, synthesis of structural protein requires that the viral DNA be transcribed to mRNA and that the mRNA be translated to generate the structural protein. For the viral model, it was assumed that such reactions could be combined together and characterized using a single-rate parameter. The lumped reactions are represented by arrows in Fig. 1.

Further assumptions made were: (1) other components necessary for the reactions to occur (i.e. nucleic acids, amino acids, etc.) were available at a constant level, (2) once a cell was infected, it could not be re-infected, even if the initial infection was cleared (i.e. superinfection could not occur), (3) reactants were distributed in a spatially homogenous manner, (4) a cell was initially infected with a pre-set number of viral templates, and (5) cells were terminally differentiated and did not divide.

\section{Deterministic Analysis}

Based on the network established in Fig. 1, a set of coupled ordinary differential equations
(ODE) was extracted:

$$
\begin{aligned}
& \frac{\mathrm{d}[\text { tem }]}{\mathrm{d} t}=k_{1}[\text { gen }]-k_{2}[\text { tem }], \\
& \frac{\mathrm{d}[\text { gen }]}{\mathrm{d} t}=k_{3}[\text { tem }]-k_{1}[\text { gen }]-k_{4}[\text { gen }][\text { struct }], \\
& \frac{\mathrm{d}[\text { struct }]}{\mathrm{d} t}=k_{5}[\text { tem }]-k_{6}[\text { struct }]-k_{4}[\text { gen }][\text { struct }] .
\end{aligned}
$$

By using an ODE model to describe viral kinetics, an underlying assumption is made that viral components change continuously with time (Érdi \& Tóth, 1988). Such an assumption is justified by assuming that the modeling results represent the average behavior of a large number of infected cells.

\section{Parameter Selection}

We assumed that gen was produced at 1 molecule cell ${ }^{-1} \mathrm{day}^{-1}$, the synthesis parameter of structural protein was 1000 -fold greater than gen, and the rate of virus production was 1 molecule tem $^{-1}$ cell $^{-1}$ day $^{-1}$. A steady-state constraint of 20 tem molecules was applied. By setting the steady state of gen and struct to 200 molecules and 10000 molecules, respectively, we determined all of the parameter values for the model, as listed in Table 1.

\section{Linear Stability Analysis}

Two steady states were calculated for the viral network. One steady state occurred when tem $=$ gen $=$ struct $=0$, the so-called "trivial" solution. The other steady state occurred at the

TABLE 1

Model parameters

\begin{tabular}{ll}
\hline Parameter & Value \\
\hline$k_{1}$ & $0.025 \mathrm{day}^{-1}$ \\
$k_{2}$ & $0.25 \mathrm{day}^{-1}$ \\
$k_{3}$ & $1.0 \mathrm{day}^{-1}$ \\
$k_{4}$ & $7.5 \times 10^{-6} \mathrm{molecules}^{-1} \mathrm{day}^{-1}$ \\
$k_{5}$ & $1000 \mathrm{day}^{-1}$ \\
$k_{6}$ & $1.99 \mathrm{day}^{-1}$ \\
\hline
\end{tabular}


values specified by the parameter constraints that were implemented: $t e m=20$, gen $=200$, struct $=10000$.

The trivial steady state was unstable, and the non-trivial steady state was stable, as determined by linear stability analysis (see Appendix A). The trivial steady state occurred when the levels of all components dropped to zero. Obviously, the virus could not propagate spontaneously. Since the infection was a perturbation from the trivial unstable steady state, the virus always reached the stable steady state under the deterministic simulation.

\section{Kinetic Regulation of Viral Synthesis}

Integration of the equations yielded switching behavior between the template amplification pathway and the virion secretion pathway. Initially, due to the lack of structural proteins, the template amplification pathway was dominant. As the level of structural protein increased, the major flux pathway switched from template amplification to virion production/secretion, illustrated in Fig. 2. In effect, the template amplification pathway is down-regulated simply through the interactions of the system.

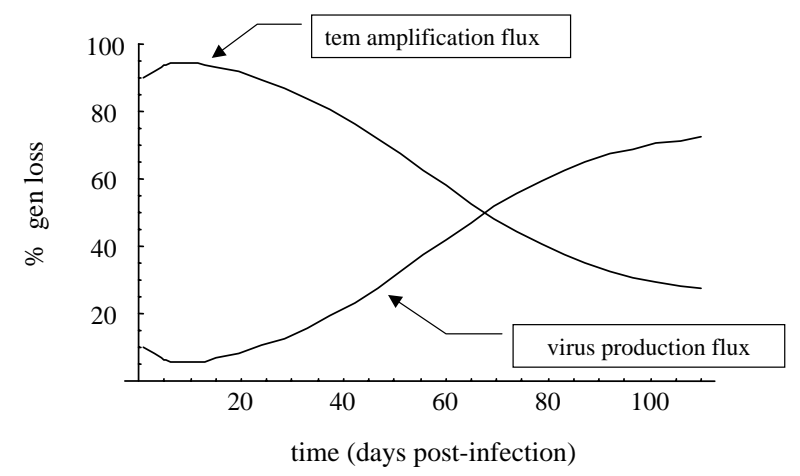

FIG. 2. Kinetics of viral replication. The switch between template amplification and virus production can be observed by plotting the output fluxes of gen. The output fluxes are normalized to total gen loss, which is defined as $\left(k_{1} \times\right.$ gen $+k_{4} \times$ gen $\times$ struct $)$. The tem amplification flux is calculated as $\left(k_{1} \times\right.$ gen $) /($ total gen loss $)$. The virus flux is calculated as $\left(k_{4} \times\right.$ gen $\times$ struct $) /($ total gen loss $)$. Using mass action kinetics as means of regulation, the virus initially amplifies its genome. As struct accumulates, virus production becomes dominant, effectively down-regulating template amplification.

\section{Stochastic Analysis}

Use of stochastic kinetic analysis provides certain advantages over deterministic analysis. When modeling chemical and biochemical species as discrete rather than continuous entities, random fluctuations that might affect reaction dynamics can be accounted for (McAdams \& Arkin, 1999). These effects may become important as the levels of reactants in a system become smaller. For example, one virus may initiate the infection of an entire organism (Jilbert et al., 1996). In such a situation, random fluctuations may impact the time evolution of the infection. The variation that arises at the cellular level can lead to a bifurcation in the cellular population, resulting in groups that behave differently depending on infection (Arkin et al., 1998). The disadvantage of a stochastic kinetic analysis is that it can be computationally expensive, making such implementations impractical or impossible.

Simulations of the stochastic kinetics were accomplished by using an event-driven continuous-time Markov process algorithm. In a Markov process, the change of a system from one state to another depends only on the current state of the system. For chemical systems, the term state refers to the amount of chemical species present. In such a context, a change of state refers to a reaction occurring, assuming no components are introduced or removed. It has been proven that elementary chemical reactions, where no reaction lumping is involved, are Markov processes (Kurtz, 1972). For our studies, we assumed that lumped reactions could also be modeled as Markov processes.

The algorithm we employed was equivalent to the Next Step method of the Gillespie Algorithm (Gillespie, 1976) as well as the method of stochastic Petri nets (Marsan et al., 1995; Goss \& Peccoud, 1998, 1999; Srivastava et al., 2001), and it proceeds as follows:

1. Calculate the potential time at which each possible reaction will occur.

2. From the list of times calculated, allow only the fastest reaction to actually occur.

3. Update the number of the reactant and product molecules appropriately. 
4. Increment the system by the amount of time it took the fastest reaction to occur.

\section{Return to step 1.}

The events are allowed to continue until a termination criterion, such as a pre-determined time, is met.

The potential time at which a reaction occurs is calculated from a weighted negative exponential density function. The function has the memory-less property (i.e. not dependent any previous states) required for a Markov process (Marsan et al., 1995), and has been shown to be the appropriate function for modeling chemical reactions (Gillespie, 1976).

Like the deterministic implementation, an assumption with the stochastic implementation was that any two reactants of a given species were indistinguishable and equally likely to be available. However, unlike the deterministic implementation, the reactants in the stochastic simulation were assumed to interact as discrete entities. Furthermore, each stochastic simulation represented the infection of an individual cell by the virus. For the deterministic implementation, the simulation represented the average behavior of a population of infected cells.

To explore whether fluctuations had any impact on the dynamics of intracellular viral replication, stochastic simulations were performed for a 200-day simulation period using the network shown in Fig. 1. Thus, viral intracellular kinetics were simulated both stochastically and deterministically using the same model framework.

\section{Individual Stochastic Results}

Using the stochastic algorithm, viral kinetics were monitored for individual cells. Examples of typical stochastic simulation results for tem are shown in Fig. 3. Each of the simulations in Fig. 3

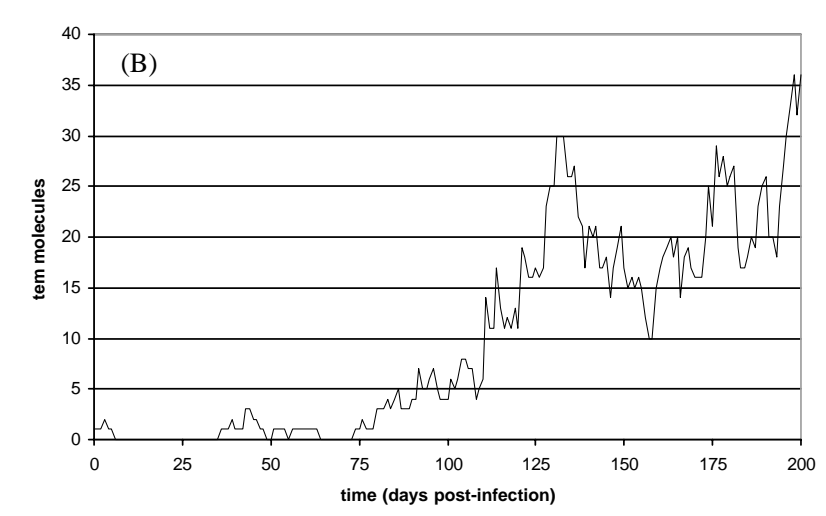

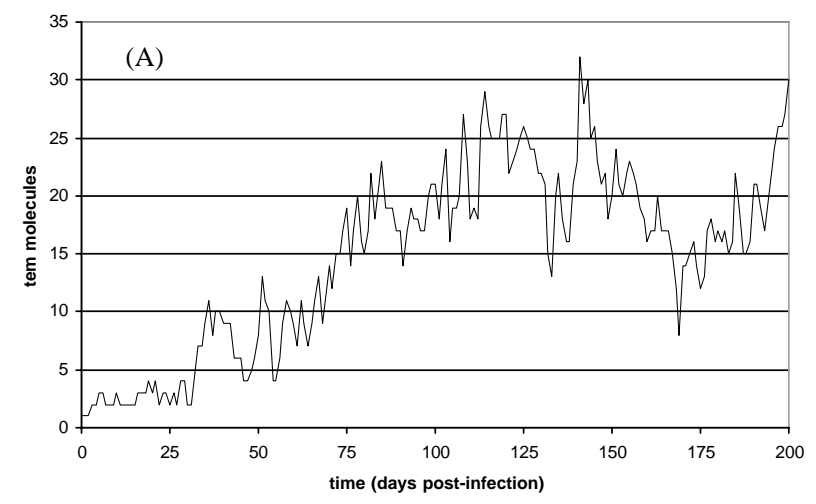

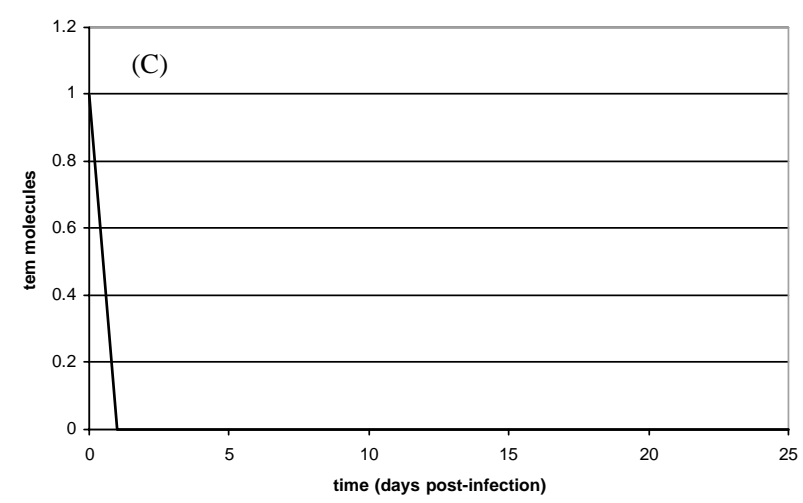

FIG. 3. Individual stochastic simulations for template (tem). Results for three typical simulations of tem are shown. In each case, the simulated infection is initiated with one molecule of tem. (a) tem steadily accumulates throughout the course of the infection to finally hover about the steady state of 20 tem molecules. (b) After an initial rise, during which gen is made, the amount of tem drops to zero and remains there for a large portion of the infection. Eventually, the gen is used to make new tem. The infection only breaks away from the unstable node once more than four molecules of tem are present. (c) tem is immediately degraded resulting in the loss of the infection. 
was initiated with one molecule of tem. Figure 3(a) represents a typical time evolution of tem dynamics. The amount of tem in the cell generally rose until it fluctuated about the steady state of 20 molecules. The structural protein and gen behaved similarly, increasing until they fluctuated around their respective steady states (data not shown). Figure 3(b) is interesting in that the tem level dropped to zero and then recovered. Such a loss and recovery occurred when the tem first synthesized gen, and then the tem was degraded. At some point, the gen was converted into new tem, allowing the virus to recover. Figure 3(c) shows that the system reached and stayed at the unstable steady state. Thus, the ability to reach the unstable steady state was attributed to two factors. First, because the reactants were discrete entities, it was not possible to have a fraction of an entity; degradation of a single entity occurred instantaneously and completely. Second, the order of events was important.

\section{Comparison of Deterministic and Stochastic Results}

Since the results of the deterministic simulation could be considered as the average viral kinetic behavior (Zheng \& Ross, 1991), they could be compared to the average results of multiple stochastic simulations. Additionally, variance data could be calculated for the viral components through statistical analysis of the stochastic runs. The average of 3000 individual stochastic simulations was compared to the deterministic simulation in the results described below. Simulations for low and high multiplicity of infection (MOI) were studied. An initial infection with one molecule of tem was chosen to represent the low MOI case. The high MOI case was represented as an initial infection of five molecules of tem, unless otherwise indicated.

The kinetics of the deterministic and averaged stochastic simulations differed significantly for the low MOI case as shown in Fig. 4. The deterministic simulation converged to the pre-set stable steady state of 20 tem molecules, whereas the stochastic simulation fell substantially short, reaching a plateau of 15 molecules of tem. The stochastic low MOI simulation results were calculated to be within $7.5 \%$ of the true mean with a confidence of $95 \%$.

Due to the discrete nature of the fluctuations during the individual runs, it was possible for the levels of viral species to "jump" to the unstable node and remain there. As a result, some of the simulations went to zero or aborted, indicating virus that was unable to establish a successful infection. Averaging the aborted infection simulations with the successful infection simulations

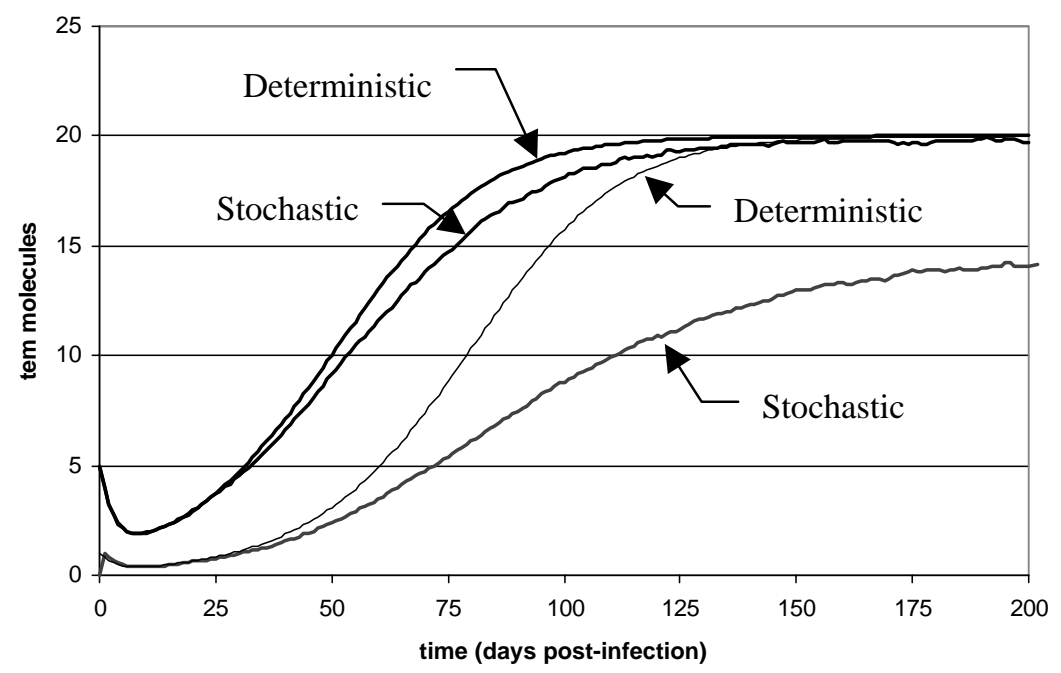

FIG. 4. Comparison of deterministic vs. average stochastic template kinetics. Results are shown for low (1 tem molecule infection) and high infection levels ( 5 tem molecule infection). Stochastic results represent the average of 3000 simulations. All stochastic results were calculated to be within at least $7 \%$ of the true mean with $95 \%$ confidence. 


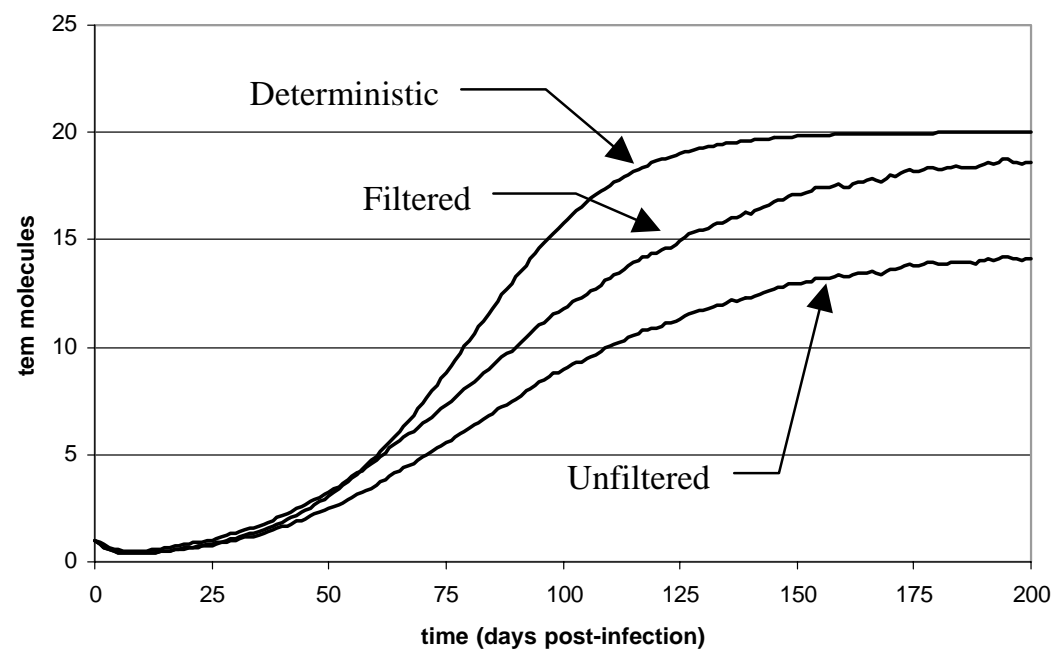

FIG. 5. Filtered low infection stochastic. The filtered results are the low infection stochastic simulations filtered of aborted simulations. An aborted simulation was defined as when both tem and gen reached zero at any point during the simulation. When filtered, the stochastic simulations approach the predicted deterministic stable steady state. However, the transient dynamics continue to differ from the deterministic results.

resulted in a value less than that predicted by the deterministic model, shown in Fig. 4. The deterministic model always had reactants available for every reaction, resulting in no possibility of the system going to zero, based on the linear stability analysis. For example, if only 0.1 molecules of tem were available per cell, a physical interpretation is that $10 \%$ of the cell population had one molecule of tem while the other $90 \%$ had none. However, from a purely mathematical perspective, a valid interpretation is that $100 \%$ of the cells had 0.1 molecules of tem each. Reactions were carried out with fractions of molecules, and although they could come very close to the unstable steady state, they could never reach it. Rather they would eventually reach the stable steady state.

Upon filtering out the unsuccessful infections, the stochastic average of the successful infections resulted in a plateau at the deterministically predicted stable node. The dynamics are shown in Fig. 5, where the filtered results are the average of 2173 successful infections. However, as will be further discussed, the transient dynamics continued to differ.

Cells infected with five molecules of tem approached the stable node regardless of the simulation method. The only way the simulation could abort was if all of the tem was degraded

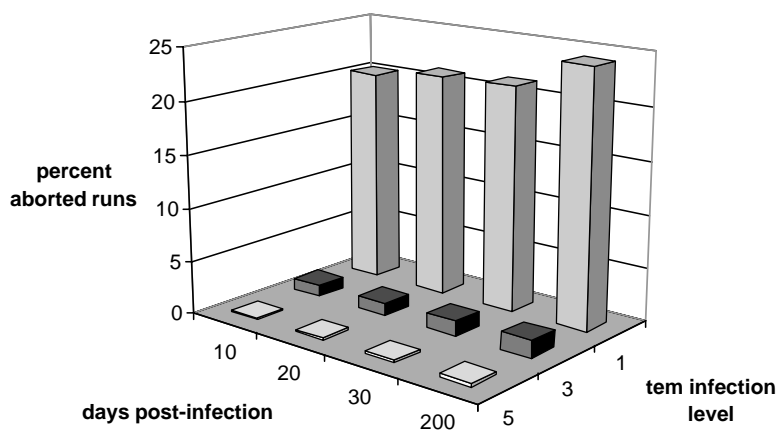

FIG. 6. Aborted simulation. The percentage of simulations aborted at differing times based on differing tem infection levels are plotted. An aborted run was defined as when both tem and gen were zero.

and none of the gen was available for tem synthesis, as was the case for the low MOI scenario. With five molecules of tem present initially, such a scenario was highly unlikely for the given parameter set. In general, increasing the MOI from one resulted in a drastic reduction of aborted simulations. With an MOI of three, only $1.6 \%$ of the simulations aborted after 200 days, as shown in Fig. 6. Increasing the MOI to five further decreased aborted infections to $0.3 \%$.

If the unstable node was accessed at all, it was generally accessed early in the infection as 
indicated in Fig. 6. For infections with an MOI of one, $20 \%$ of the simulations aborted within 10 days. Only an additional $4 \%$ failed for a 200 -day simulation. For the other MOIs evaluated, there were no appreciable changes in the number of the aborted runs after the first 10 days. Essentially, once more than one tem was formed, it became highly unlikely that all the tem would be degraded before any gen was formed and able to generate new tem.

\section{Analysis of Transient Kinetics}

Although the deterministic and averaged stochastic simulations approached the same steady state if all of the aborted runs were filtered out, a statistically significant difference remained between the dynamics of the deterministic and average stochastic results as determined by the Z-test (Walpole \& Myers, 1989). When initiated with five molecules of tem, the deterministic and the average stochastic simulations converged to the stable steady state as determined from the deterministic model. However, the stochastic result underestimated the deterministic result during the transient portion of the simulation, when filtered. To make sure that the result was not simply due to running very few simulations, a 95\% confidence interval analysis was performed. The averaged result of the stochastic high MOI simulation was calculated to be within $3.2 \%$ of the true mean with $95 \%$ confidence. Thus, the difference between the average stochastic results and the deterministic results were statistically significant.

To test whether the differences seen were due to the increasing dominance of the nonlinear term, deterministic and stochastic simulations were performed with $k_{4}$ set to zero, effectively removing the nonlinear portion of the model. Under this scenario, the deterministic and the averaged stochastic results for the five-molecule simulation matched exactly (data not shown). This result suggested that the nonlinearity of the model did indeed contribute to the difference in the trajectories. Further corroboration was inferred by examining the dynamics of the full model when the nonlinear term was negligible. The negligible period occurred during the first 30 days of the simulation. From Figs 4 and 5, we noticed that there was little to no disparity among the different modeling results.

\section{Distribution of Intracellular Viral Components}

The standard deviation of the low MOI stochastic simulations was over twice as large as the high MOI simulation by the end of the time course (data not shown). However, initially it was the higher MOI that had the larger standard deviation. To understand why such a difference occurred, it was useful to analyse the actual distributions.

Although at the onset of infection there was a slight dip in the level of tem in all cases due to initial degradation (see Fig. 4), generally enough gen was made that tem could be replaced through synthesis. Furthermore, the level of tem in the high MOI case did not fall as much as it did for the low MOI case. The high MOI cells were able to rapidly progress to the stable node. For a very few individual simulations, the level of tem fell very close to zero and remained near there for 150 days or more, which can be seen in Fig. 7. Typically, however, simulations in which viral component levels were low for an extended period of time behaved in the manner depicted in Fig. 3(b). In these situations, gen was shunted through the virion production pathway rather than the tem synthesis pathway. Eventually, tem was amplified to the point where the degradation of the occasional tem molecule did not significantly impact production of the other viral components. As a result, the driving force was overwhelmingly toward the stable node.

The distribution of tem for the low MOI case showed that even when runs were not aborted, a substantial number of individual simulations remained near the unstable node, seen in Fig. 8. It should be noted that only simulations that went to completion (i.e. did not abort within the 200-day time period) are included in the distributions shown in Fig. 8. There was much slower movement of the population towards the stable node as compared to the high MOI case. The attraction and hovering near the unstable node provided a further reason why the averaged stochastic simulations showed slow dynamics in approaching the stable node. A portion of the viral population simply continued to hover near 

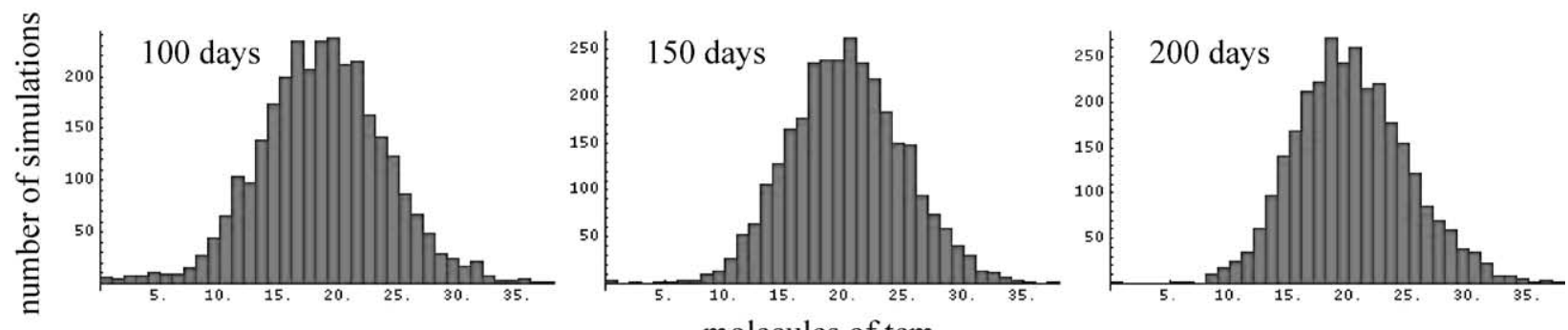

FIG. 7. Unfiltered high infection template distribution. The raw tem frequency distribution for the high unfiltered infection cases are shown at 100,150, and 200 days post-infection. The $x$-axis indicates the number of tem molecules present in a cell; the $y$-axis indicates the number simulations possessing the given number of tem molecules. In each of the cases shown, the stochastic average value and deterministic value are identical.

the zero level, bringing down the average value of the viral components. During the 100-200day period, the low MOI distribution was bimodal in appearance. The high standard deviation was due to the large spread of the viral components. As the simulation progressed, however, the distribution slowly migrated from the unstable node to the stable node.

\section{Discussion}

Our results illustrated how the properties of a stochastic model could lead to a different behavior from that of a deterministic model for intracellular kinetics that initially involved a low number of reactants. Since infections could be initiated by a single virion, the "low number of reactants" criteria could easily be met.

Part of the reason that the deterministic and stochastic model results differed was due to the assumption of continuity of biochemical species in the deterministic model. It has been assumed that by looking at a large number of cells, the continuity assumption across biochemical species holds. However, this assumption may be misleading. The segregation of these intracellular species within cells discretizes the system. The result of such segregation is a population distribution of species on a per cell basis rather than a well-mixed homogenous system across cells.

Whether or not a natural infection takes hold in a cell may be a matter of chance. With the deterministic simulation, however, the introduction of a virus genome in a cell irreversibly progressed to yield virus progeny. The stochastic implementation of the same model with the same parameters allowed access to the unstable steady state, illustrating how stochastic fluctuations could affect viral replication.

It was interesting to note the differences in the transient kinetics of the deterministic simulations and the stochastic simulations, regardless of whether aborted runs were included. Such differences can have an impact on development and characterization of anti-virals, particularly when the reaction network is nonlinear. Fitting the deterministic simulation and the stochastic simulation results to match up with experimental results, for example, would require the use of different parameters depending upon the modeling methodology. If kinetic parameters of the anti-viral were characterized based on the model, the question of what parameter set to use then arises. Such information is particularly critical if the kinetic parameters of the anti-viral will be used in the development of a treatment strategy.

Our results suggested an interesting hypothetical mechanism for viral persistence. If the immune system of the host were only able to mount a response to cells infected above a particular threshold level, then cells harboring virus below the threshold could serve as longterm reservoirs. From our model, we were clearly able to stochastically simulate a bimodal population of infected cells, allowing for the possibility of such a reservoir, given the constraints of our model. If such a population is evolutionarily favored in nature, infection by a large number of viruses may not be optimal.

Although stochastic modeling, and modeling in general, has the potential to provide new insights into biological processes, it is necessary 

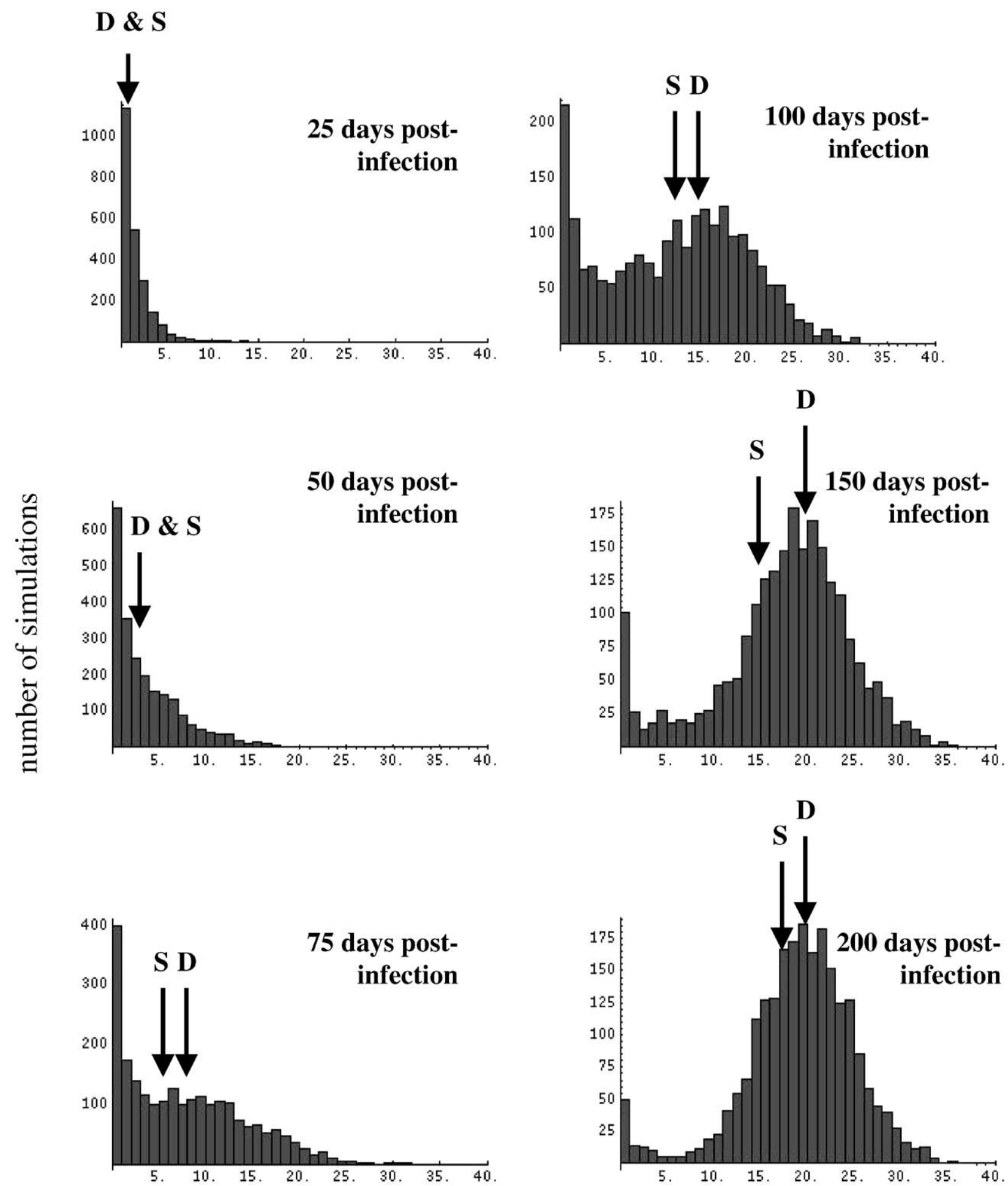

\section{molecules of tem}

FIG. 8. Filtered low MOI tem distribution. The raw tem frequency distributions for the low filtered infection cases are shown. The $x$-axis indicates the number of tem molecules present in a cell; the $y$-axis indicates the number simulations possessing the given number of tem molecules. The $\mathbf{S}$ refers to the average stochastic value, while the $\mathbf{D}$ refers to the value calculated via the deterministic simulation. 
to be cognizant of the caveats that come with such an analysis. One such caveat to be aware of in our model was that many complex reactions were combined or lumped (e.g. transcription and translation steps, etc.) into one reaction. As a first pass, we felt such an assumption was justifiable to determine overall behavior of our network. However, as more detailed questions about the network are asked, it will be necessary to either "un-lump" key reactions, or determine in a rigorous and quantitative manner the effect that lumping has upon simulation results. Additionally, we assumed that all reactants within the cell were spatially homogenous. Once again, we felt such an assumption was justifiable given the questions we were investigating. For more specific biological questions, spatial heterogeneity will need to be addressed, especially given the importance of processes such as compartmentalization and localization in many biological systems.

Stochastic implementations of virus models challenge us to rethink how we formulate new anti-viral strategies. We have previously employed a kinetic model for the intracellular growth of a virus to show that drugs targeting components of negative-feedback loops can gain effectiveness against mutant viruses that attenuate the drug-target (Endy \& Yin, 2000). Such a strategy creates, in essence, an evolutionary barrier that selects against observed modes of anti-viral escape, but it is based on a deterministic rendering of the intracellular kinetics. Stochastic kinetic mechanisms generate many trajectories that together may define two or more classes of different dynamic behaviors, as we have seen here: low-multiplicity infections yielded both high- and low-activity host-cell producers. It remains an open question how drug-target interactions, which may themselves exhibit significant fluctuations, influence the integrated dynamics of virus growth.

This work was supported by the National Institute of Health Cancer Biology Training Grant (T32 CA09075) and the Quantitative Systems Biotechnology Program (BES-0120361) of the National Science Foundation. Support for J.S. was provided by the National Institute of Health (CA84017).

The authors would also like to thank Dr. James B. Rawlings for helpful discussions.

\section{REFERENCES}

Arkin, A., Ross, J. \& McAdams, H. H. (1998). Stochastic kinetic analysis of developmental pathway bifurcation in phage lambda-infected Escherichia coli cells. Genetics 149, 1633-1648.

Chin, T. M. \& Willsky, A. S. (1989). Stochastic Petri net modeling of wave sequences in cardiac arrhythmias. Comput. Biomed. Res. 22, 136-159.

ENDY, D. \& YIN, J. (2000). Toward antiviral strategies that resist viral escape. Antimicrob. Agents Chemother. 44, 1097-1099.

Endy, D., Kong, D. \& Yin, J. (1997). Intracellular kinetics of a growing virus: a genetically structured simulation for bacteriophage T7. Biotechnol. Bioeng. 55, 375-389.

Endy, D., You, L., Yin, J. \& Molineux, I. J. (2000). Computation, prediction, and experimental tests of fitness for bacteriophage T7 mutants with permuted genomes. Proc. Natl Acad. Sci. U.S.A. 97, 5375-5380.

Érdi, P. \& Tóth, J. (1988). Mathematical Models of Chemical Reactions : Theory and Applications of Deterministic and Stochastic Models. Princeton, NJ: Princeton University Press.

GillesPiE, D. T. (1976). A general method for numerically simulating the stochastic time evolution of coupled chemical reactions. J. Comput. Phys. 22, 403-434.

Goss, P. J. \& Peccoud, J. (1998). Quantitative modeling of stochastic systems in molecular biology by using stochastic Petri nets. Proc. Natl Acad. Sci. U.S.A. 95, 6750-6755.

Goss, P. J. \& Peccoud, J. (1999). Analysis of the stabilizing effect of Rom on the genetic network controlling ColE1 plasmid replication. In: Pacific Symposium on Biocomputing, Mauna Lani, Hawaii, pp. 65-76.

Jilbert, A. R., Miller, D. S., Scougall, C. A., TurnBull, H. \& Burrell, C. J. (1996). Kinetics of duck hepatitis $\mathrm{B}$ virus infection following low dose virus inoculation: one virus DNA genome is infectious in neonatal ducks. Virology 226, 338-345.

Knipe, D. M. \& Howley, P. M. (eds). (2001). Fields Virology. Baltimore, MD: Lippincott Williams \& Wilkins Publishers.

KuRTZ, T. G. (1972). The relationship between stochastic and deterministic models for chemical reactions. J. Chem. Phys. 57, 2976-2978.

Marsan, M. A., Balbo, G., Conte, G., Donatelli, S. \& Franceschinis, G. (1995). Modelling with Generalized Stochastic Petri Nets. Chichester: John Wiley \& Sons.

McAdams, H. H. \& Arkin, A. (1997). Stochastic mechanisms in gene expression. Proc. Natl Acad. Sci. U.S. A. 94, 814-819.

McAdams, H. H. \& Arkin, A. (1999). It's a noisy business! Genetic regulation at the nanomolar scale. Trends Genet. 15, 65-69.

REDDY, B. \& YIN, J. (1999). Quantitative intracellular kinetics of HIV Type 1. AIDS Res. Human Retroviruses 15, 273-283.

Srivastava, R., Peterson, M. S. \& Bentley, W. E. (2001). Stochastic kinetic analysis of the Escherichia coli stress circuit using sigma(32)-targeted antisense. Biotechnol. Bioeng. 75, 120-129.

Walpole, R. E. \& Myers, R. H. (1989). Probability and Statistics for Engineers and Scientists. New York: Macmillan Publishing Company. 
You, L. \& Yin, J. (2000). Patterns of regulation from mRNA and protein time-series. Metab. Eng. 2, 210-217. You, L. \& YIN, J. (2001). Simulating the growth of viruses. In: Pacific Symposium on Biocomputing (Altman, R. B., Dunker, A. K., Hunter, L., Lauderdale, K. \& Klein, T. E. (eds)), Singapore: World Scientific.

You, L., Suthers, P. \& Yin, J. (2002). Effects of Escherichia coli physiology on the growth of phage T7 in vivo and in silico. J. Bacteriol. 184, 1888-1894.

ZHenG, Q. \& Ross, J. (1991). Comparison of deterministic and stochastic kinetics for nonlinear systems. J. Chem. Phys. 94, 3644-3648.

\section{Appendix A}

\section{Linear Stability Analysis}

Given a system of differential equations of the form

$$
\frac{\mathrm{d} V_{i}}{\mathrm{~d} t}=f_{i}\left(V_{1}, V_{2}, V_{3}, \ldots, V_{n}\right),
$$

any steady state can be written as

$$
0=f_{i}\left(\tilde{V}_{1}, \tilde{V}_{2}, \tilde{V}_{3}, \ldots, \tilde{V}_{n}\right),
$$

where $\tilde{V}$ is the steady-state value of $V$.

Let

$$
\varepsilon_{i}=V_{i}-\tilde{V}_{i}
$$

Performing a Taylor series expansion about the steady-state values of $f_{i}$ results in

$$
\begin{aligned}
& f_{i}\left(V_{1}, V_{2}, V_{3}, \ldots, V_{n}\right)=f_{i}\left(\tilde{V}_{1}, \tilde{V}_{2}, \tilde{V}_{3}, \ldots, \tilde{V}_{n}\right) \\
& +\sum_{j=1}^{n} \beta_{i j} \varepsilon_{j}+\text { higher order terms, }
\end{aligned}
$$

where

$$
\beta_{i j}=\left.\frac{\partial f_{i}}{\partial V_{j}}\right|_{V_{k}=\tilde{V}_{k}}
$$

Taking the derivative of eqn (A.4) results in

$$
\frac{\mathrm{d} \varepsilon_{i}}{\mathrm{~d} t}=\frac{\mathrm{d} V_{i}}{\mathrm{~d} t}-\frac{\mathrm{d} \tilde{V}_{i}}{\mathrm{~d} t} .
$$

However, at steady state, $\mathrm{d} \tilde{V}_{i} / \mathrm{d} t=0$. Using eqn (A.1), it is possible to re-write eqn (A.5) as

$$
\frac{\mathrm{d} \varepsilon_{i}}{\mathrm{~d} t}=\frac{\mathrm{d} V_{i}}{\mathrm{~d} t}=f_{i}\left(V_{1}, V_{2}, V_{3}, \ldots, V_{n}\right) .
$$

If the higher order terms of eqn (A.4) are neglected, eqn (6) can be written as

$$
\begin{aligned}
\frac{\mathrm{d} \varepsilon_{i}}{\mathrm{~d} t} \approx f_{i}\left(\tilde{V}_{1}, \tilde{V}_{2}, \tilde{V}_{3}, \ldots, \tilde{V}_{n}\right) \\
+\sum_{j=1}^{n} \beta_{i j} \varepsilon_{j} .
\end{aligned}
$$

However, since $f_{i}$ is being evaluated at its steady state in eqn (A.7), from eqn (A.2), we know that $f_{i}$ must be 0 . So

$$
\frac{\mathrm{d} \varepsilon_{i}}{\mathrm{~d} t} \approx \sum_{j=1}^{n} \beta_{i j} \varepsilon_{j} .
$$

The general solution for this system of equations is

$$
\varepsilon_{i} \approx \sum_{j=1}^{n} A_{i j} \mathrm{e}^{\lambda_{j} t}
$$

where $A_{i j}$ is the constant of integration and $\lambda_{j}$ is the eigenvalue of the stability matrix. Equation (A.8) can be written in matrix form as

$$
\left[\begin{array}{cccc}
\beta_{11} & \beta_{12} & \ldots & \beta_{1 n} \\
\beta_{22} & & & \vdots \\
\vdots & & & \vdots \\
\beta_{n 1} & \ldots & \ldots & \beta_{n n}
\end{array}\right]
$$

where eqn (A.10) is the stability matrix. It is also the Jacobian of the system of differential equaitons. Equation (A.10) can be re-cast as

$\operatorname{Det}\left[\begin{array}{cccc}\beta_{11}-\lambda_{1} & \beta_{12} & \ldots & \beta_{1 n} \\ \beta_{22} & & & \vdots \\ \vdots & & & \vdots \\ \beta_{n 1} & \ldots & \ldots & \beta_{n n}-\lambda_{n}\end{array}\right]=0$

to determine the eigenvalues.

From eqn (A.9), it can be seen that if $\lambda$ is negative, the solution to $\varepsilon$ goes to zero. Based on the definition of $\varepsilon$ from eqn (A.3), it means any perturbation from $\tilde{V}$ comes back to $\tilde{V}$. Thus, $\tilde{V}$ is a stable steady state. If $\lambda$ is positive, $\varepsilon$ goes to infinity. Hence, any perturbation from $\tilde{V}$ results 
in $V$ moving away from the steady state, resulting in $\tilde{V}$ being classified as an unstable steady state. If $\lambda$ is complex, then $V$ will either approach or veer away from $\tilde{V}$ in an oscillatory manner depending on the sign of the real part of $\lambda$.

Applying the above procedure to the deterministic generic virus model, described by eqn (1), resulted in two steady states. One steady state occurred at

$$
\begin{aligned}
& \text { tem }=0, \\
& \text { gen }=0, \\
& \text { struct }=0
\end{aligned}
$$

and the other occurred at

$$
\begin{aligned}
& \text { tem }=20, \\
& \text { gen }=200, \\
& \text { struct }=10000 .
\end{aligned}
$$

The Jacobian of eqn (1) was calculated to be $\left[\begin{array}{ccc}-k_{2} & k_{1} & 0 \\ k_{3} & -k_{1}-k_{4} \text { struct } & -k_{4} g e n \\ k_{5} & -k_{4} \text { struct } & -k_{6}-k_{4} \text { gen }\end{array}\right]$.
Using Mathematica 4.1, the eigenvalues for the steady state described by eqn (A.12) were calculated to be

$$
\begin{aligned}
& \lambda_{1}=-0.3315 \\
& \lambda_{2}=0.0565, \\
& \lambda_{3}=-1.9985 .
\end{aligned}
$$

The eigenvalues for the steady state described by eqn (A.13) were

$$
\begin{aligned}
& \lambda_{1}=-2.0113-2.7756 \times 10^{-17} i, \\
& \lambda_{2}=-0.0691+1.1102 \times 10^{-16} i, \\
& \lambda_{3}=-0.2696-1.1102 \times 10^{-16} i .
\end{aligned}
$$

The presence of a positive eigenvalue in eqn (A.15) denoted that the steady state described by eqn (A.12) was unstable. Likewise, since all of the eigenvalues in eqn (A.16) were negative, the steady state described by eqn (A.13) was stable. It should be noted that, based on the order of magnitude, the imaginary components of eqn (A.16) are likely an artifact of the numerical solver algorithm implemented in Mathematica. Phase plane plots confirmed the stability of the steady state described by eqn (A.13) (data not shown) and resulted in no oscillations. 